\title{
Ein ohne Bewusstseinstrübung ablanfendes paralytisches Äquivalent der genuinen Epilepsie in Form von Status hemiparalyticus. \\ Von
}

Heinrich Higier-Warschau.

Die meisten Autoren betonen, mit wie grosser Reserve die Diagnose „Epilepsie“ zu stellen sei bei epileptoiden, ohne Bewusstseinsverlust vor sich gehenden Insulten, da echt epileptische Ereignisse, sei es auf motorischem, sensiblem, sensorischem oder psychischem Gebiet, bei erhaltenem Bewusstsein etwas-sehr Seltenes sein sollen. Noch schwerer ist diese Diagnose zu stellen bei Fehlen jeder Aura und postparoxysmaler Missempfindungen.

Was diejenige Varietät anbelangt, die man Status epilepticus zu nennen pflegt, so gehört sie bekanntlich zu den schwersten Formen der Fallsucht, nur fehlt es leider z. Z. an einer präcisen Definition derjenigen Zustände, die wir noch Anfallserien oder schon Status epilepticus mit der landläufigen infausten Prognose nennen dürfen. Es gibt eben Anfallserien, die in den Status. im engeren Sinne übergehen können. Bei den meisten Definitionen bildet die andauernde Bewusstlosigkeit oder das Koma zwischen den einzelnen Anfällen das Hauptunterscheidungsmerkmal. Gowers rechnet auch Anfallserien, in deren Zwischenpausen das Bewusstsein unvollkommen erwacht, zum Status.

Donner meint, die Beurteilung der Schwere des Status von dem Verhalten des Herzens abhängig machen und alle Anfallserien von diesem Gesichtspunkte aus als leichte, mittelschwere und schwere Formen unterscheiden zu müssen. Die leichte Form bestände demnach in Anfallserien mit grösseren Zwischenpausen, in denen das Bewusstsein ganz oder zum Teil erhalten ist und der Puls in den Anfallpausen annähernd normal bleibt. Die mittelschwere Form bestände in Anfallserien mit schweren Anfüllen, die häufig aufeinander folgen, mit grösstenteils geschwundenem Bewusstsein in den Pausen, doch ohne ernstere Herzstörung, Pulsbeschleunigung in der Anfallpause bis höchstens 110-120 in der Minute. Bei der schweren Form folgen sich die Anfälle häufig, Störung der Herzfunktion zeigt sich in kleinem 
oder unregelmässigem Puls oder Beschleunigung von 120-180 und mehr in der Minute während der Anfallpause. „Also nicht die Schwere und Häufigkeit der Anfälle allein sollte für die Beurteilung ausschlaggebend sein, sondern auch das Verhalten des Herzens den Attacken gegeniiber in Betracht gezogen werden."

Nach anderen Kennern der Epilepsie wird jede „Anfallserie“ zur schweren Form des „Status" viel mehr durch die erböhte Körpertemperatur, als durch den völligen interparoxysmalen Bewusstseinschwund eder die gesteigerte Herzaktion gestempelt.

War man sich somit über die Definition des Status epilepticus einigermassen klar, so fehlte es noch bis vor einem Decennium an Übereinstimmung der Kliniker über die Daseinsberechtigung der halbseitigen Form des Status bei der genuinen Epilepsie. Wie aus der Zusammenstellung L. Müllers aus der Nonneschen Abteilung zu ersehen ist, glaubte z. B. Nothnagel, ein ausgezeichneter Bearbeiter der Epilepsie, nicht an die Halbseitigkeit des Morbus sacer, und Binswanger, späterer Monographist der Fallsucht, dem die Jacksonsche Form bei genuiner Fallsucht nicht fremd war, wollte dennoch nicht zubilligen die Existenzberechtigung des halbseitigen genuinen Status epilepticus, des von Winkler zuerst erschöpfend beschriebenen Status epilepticus unilateralis. Dasselbe gilt von Gowers, einem der besten Kenner der Epilepsie. Féré, der den idiopathischen lokalisierten Status epileptieus zugibt, vindiziert ihm jedoch mit Unrecht gegenüber der allgemeinen eine auffallende Benignität, was sich jedoch, wie spätere Arbeiten bewiesen haben, keineswegs bestätigte.

Erst Müller und Stertz konnten speziell im Anschluss an französische Autoren (Landouzy et Siredey, Pic et Piéry) einzelne überzeugende Beispiele dieser exzeptionellen Form aus der ältesten Literatur anführen und an einer grösseren Reihe diesbezüglicher letaler, antoptisch verifizierter Fälle von Status hemiepilepticus genuinus gegen die Ansicht über die Benignität Front machen. Da wir nun, meint Müller, die partielle idiopathische Epilepsie als eine der allgemeinen Epilepsie durchaus äquivalente Krankheit anerkennen, warum soll sich dann nicht anch die Hemiepilepsie einmal in der schwersten Form des Status hemiepilepticus manifestieren? Oder warum soll nicht auch die Hemiepilepsie - besonders wenn ihre schlummernde Disposition durch irgendeine toxisch oder mechanisch wirkende Schädlichkeit aus ihrer Latenz aufgestört wird - nicht sofort als "Iokalisierter" Status epilepticus einsetzen? Manche Enttäuschung bei der operativen Therapie des lokalisierten Status epilepticus wird zweifelsohne demjenigen erspart bleiben, der mit dem Symptomenbild 
Ein ohne Bewusstseinstrübung ablaufendes paralytisches Äquivalent usw. 97

des Status hemiepilepticus idiopathicus vertraut ist und deswegen die naheliegende Diagnose einer organischen Gehirnaffektion, wie Neubildung resp. intrameningeale Hämorrhagie, mit grösserer Vorsicht and Reserve stellt und danach auch ihre Therapie zurickhaltender beurteilt.

Ist somit, wie gesagt, ein Fall von Epilepsie zu den seltenen zu rechnen, insofern er ohne Bewusstseinstrübung verläuft und als Status unilateralis sich kundgibt, so wird er geradezu zur rara avis, sobald er sich anf motorischem Gebiet weder als tonischer noch als klonischer Krampf abspielt, sondern stereotyp als voriubergehende Lähmung auftritt, sozusagen, als Status hemiparalyticus idiopathicus.

Was ich unter paralytischen Äquivalenten der Epilepsie oder epileptoiden Lähmungen verstehe, habe ich seinerzeit in 2 Abhandlungen (1897 und 1899) genauer besprochen und sind analoge Beobachtungen ron späteren Autoren beschrieben worden, zuletzt von Mc. Conell (1899) und von Neurath (1912).

Das klinisché Bild des paralytischen Âquivalentes lässt sich in einigen Worten kurz skizzieren. Bei Individuen, die entweder schon vorher Jacksonsche epileptische Anfälle gezeigt haben oder erst später solche bekommen, treten in verschiedener Häufigkeit in Intervallen von Tagen, Wochen oder Monaten, aber auch mehrmals am Tage, zuweilen eingeleitet in den betroffenen Extremitäten von sehr intensiven Parästhesien, die den Patienten vom Schlafe wecken, Lähmungen auf, die meist mono- oder hemiplegisch sind und zwischen Stunden und Sekunden dauern. Die aktive Motilität wird ganz ausgeschaltet, dagegen bleiben die passive Beweglichkeit intakt, die Sensibilität, Sehnen- und Hautreflexe vollständig erhalten.

Manche Autoren wollen diese „epileptischen Lähmungen" nur bei Hirntumoren und Hirnlues beobachtet haben, was jedoch unbedingt falsch ist, wie die langjährige Katamnese meiner eigenen, vor Jahren veröffentlichten Fälle beweist.

Vielfach wurde zur Erklärung der paralytischen Äquivalente auf die Theorien rekurriert, die, auf den allgemeinen Epilepsiehypothesen fussend, der postepileptischen lähmung pathogenetisch näher kommen wollten (sog. Erschöpfungstheorie). Ohne mich auf die von mir vertheidigte, die physiologischen Experimente an Affen Sherringtons berücksichtigende Hemmungshypothese einzulassen, will ich über eine neue Beobachtung berichten, die einen älteren Herrn betrifft im Stadium vermutlicher Hirnarteriosklerose und die in vielfacher Hinsicht von meinem älteren Material klinisch abzuweichen scheint.

56 Jahre alt. Kaufmann. Hat früher nie ernstliche Krankheiten durch- 
gemacht. Potus und Lues negiert. Keine Aborte bei der Frau. Gesunde kräftige Kinder.

Vor 10 Jahren allgemeiner Krampfanfall obne nachweisbare Ursache, mit absoluter Bewusstlosigkeit, Cyanose und Schaum vor dem Munde. Vor 4 Jahren "apoplektiformer Insult" mit Verlust des Bewusstseins, der etwa eine Stunde dauerte, ohne eine Lähmung auf motorischem oder sensiblem Gebiete zu hinterlassen. Keine psychischen Äquivalente der Fallsucht. $A b$ und zu leichte Schwindelanfälle.

Eines Tages stellt sich plötzlich Lähmung des rechten Armes ein, die rasch vorübergeht, um sich darauf oft zu wiederbolen, gelegentlich auf das Bein und die Gesichtshälfte derselben Seite ubergeht und in der Regel von Sprachstörungen begleitet wird. Die Lähmung oder Schwäche wird ab und $\mathrm{zu}$ von einem prickelnden unangenehmen Gefühl in den Fingern und dex Zunge eingeleitet oder begleitet.

Der Ausfall der Motilität dauert zwischen 1 und 5 Minuten und ist meist absolut, wobei das Bewusstsein fut kein Moment getrübt bleibt. Das Gesicht nimmt selten, die Zunge nie Anteil an der Lähmung.

Der Muskeltonus ist nicht gesteigert, die Sebnen- und Hautreflexe sind normal, speziell beim permanenten oder transitorischen Babinski.

Die Sensibilität ist ungestört, die Pupillen sind nicht erweitert oder deformiert, reagieren prompt auf Licht, Konvergenz und Akkomodation wäbrend der Anfälle.

Die Lahmung beginnt meist in den Fingern, setzt sich auf den ganzen Arm fort, gelegentlich auch auf das Bein der rechten Seite obne eine Spur von tonischen Krämpfen oder klonischen Zuckungen aufzuweisen, während die linke Seite immer ganz frei bleibt. Die Anfälle folgen sich zum Teil Schlag auf Schlag, wobei auch dazwischen Pat. völlig bei Bewusstsein bleibt und erschrocken über das unangenehme Gefühl der einleitenden Parästhesien und der Dysarthrie oder kompletten motorischen Aphasie sich unterbält, bis ihn nach wenigen bis 15 Minuten aufs neue ein Anfall überstürzt.

Keine Temperatursteigerung während der Paroxysmen, nur schwankt der reguläre Puls zwischen 72 und 86 .

Von den 4 Tagen, während deren den Patienten die Anfälle quälten, waren besonders schwer die zweiten 24 Stunden, die beinahe keine einzelne Viertelstunde anfallsfrei verliefen. Das Allgemeinbefinden verschlechterte sich nicht wesentlich, der Pnls blieb regelmässig und voll, die Herzaktion ungestört und nicht beschleunigt.

Geschlängelte, rigide Radialarterien, Akzentuation des 2. Aortentones, erhőhter Blutdruck. Lungenemphysem mässigen Grades.

Urin eiweiss- und zuckerfrei, reich an Oxalsäure.

Zeichen eines sonstigen organischen Leidens, speziell Zeichen von Hirndruck fehlen ganz.

An den Augenmuskeln und am Augenhintergrund ist nichts Abnormes festzustellen.

Pat. ist geistig relativ regsam. Intelligenz ganz normal. Nichts von bysterischen Stigmen.

Vom 5. Tage an wurden immer seltener die Anfälle, um schliesslich ganz aufzuhören.

Eine weitere 10 monatliche Beobachtung hat keine Abweichung von der Norm im Nervensystem ergeben. Die Anfalle sind nicht wiedergekehrt. 
Ein ohne Bewusstseinstrübung ablanfendes paralytisches Äqujvalent usw. 99

Der besprochene Fall ist so ziemlich unkompliziert und in seinem Zustandsbilde dennoch als Unikum aufzufassen. Der 56 jährige, mit allgemeiner Arteriosklerose behaftete Herr hat in seinem Leben vor 10 Jahren einen epileptischen Krampfanfall and macht vor 4 Jahren einen schnell vorübergehenden ,apoplektiformen Insult" ohne nachfolgende Paresen, wahrscheinlich ebenfalls epileptischer Natur durch. Vor 10 Monaten bekommt er ganz unerwartet eine rechtsseitige Monoplegia superior, die nach wenigen Minuten spurlos schwindet, um sich darauf in kurzen Intervallen 4 Tage hintereinander fortwährend zu wiederholen - mehrmals in der Stunde -, hie und da die ganze Körperhälfte affizierend, beinahe regelmässig in Begleitung von Dysarthrie oder Aphasie.

Dieser Status epilepticus oder epileptiformis, wenn man ihn so nennen darf, da die Paroxysmen tagelang unaufhörsam wiederkehren, unterschied sich in mehrfacher Hinsicht von den klassischen Formen: Erstens bestand keine Bewusstseinstrübung während der Anfälle und Somnolenz in den postparoxysmalen Perioden, zweitens fehlten sämtliche Puls- und Herzstörungen oder Temperatursteigerungen während des ganzen Status, drittens generalisierten sich die Krankheitserscheinungen niemals auf den ganzen Körper, sondern blieben stets an derselben Körperhälfte lokalisiert (Status hemiepilepticus) und viertens, was das Eigentümlichste und Interessanteste darstellt, fehlten während des ganzen Status sowohl tonische als klonische Krämpfe und herrschten einzig und allein motorische Ausfallserscheinungen ohne Sensibilitätsstörungen (Status hemiparalyticus).

Dass es sich um genuine Epilepsie handelt, die möglicherweise als spät aufgetretene (E. tarda) mit Hirnarteriosklerose in losem Zusammenhang steht, geht aus der ganzen Krankengeschichte herror, die keine weitere epikritische Besprechung erfordert, da nichts in derselben - während der 10 Jahre vom 1. epileptischen Anfalle und während der 10 ruhigen Monate nach der grossen Anfallserie - für eine organische Erkrankung des Cerebrum spricht (Hirnlues, Tumor cerebri, intrameningeale event. intracerebrale Blutung).

Solche Fälle von Status hemiparalyticus epilepsiae genuinae gehören jedenfalls zu den grössten Seltenheiten und verdienen veröffentlicht zu werden. Die pathogenetischen Gesichtspunkte dieser paralytischen Äquivalente sind näher in meinen zwẹ Abhandlungen berücksichtigt worden und sollen an dieser Stelle nicht wiederholt werden. 
100 Hrgrer, Ein ohne Bewusstseinstrübung ablaufendes paralytisches usw.

\section{Literatur.}

Donner, Über den Status epilepticus und .... Zeitschrift für Psychiatrie, Bd. 69.

Féré, C. O., Note sur des attaques paralytiques chez. un épileptique. Comptes rendus hebdom. des séances et mêm. de la Soc. de Biol. 30, 1896.

Higier, H., Paroxymal auftretende Lähmungen epileptischer Natur. Neurol. Zentralbl, 4, 1897.

Derselbe, Zur Pathogenese der motorisch-paralytischen Äquivalente des epileptischen Anfalles. Deutsche Zeitschrift für Nervenheilkunde. 14, 1907.

Derselbe, Epilepsia tarda in Form von Status hemiepilepticus idiopathicus, verbunden mit Mikropsie und eigentümlichen Bewegungsempfindungen an den Extremitäten. Neurol. Zentralbl. 8, 1916.

Landouzy et Siredey, Revue de Médecine 1894.

Löwenfeld, L., Beiträge zur Lehre von der Jacksonschen Epilepsie und den klinischen Äquivalenten derselben. Archiv für Psychiatrie. Bd. 21. 1890 .

Mc. Conell, J., Transient paralysis as un epileptic equivaleut. The Journal of nerv. and ment. Dis. 26, 1899.

Müller, L., Über Status hemiepilepticus idiopathicus. Deutsche Zeit. schrift für Nervenheilkunde. 28, 1904.

Neurath, B., Paralytische Äquivalente dor Kinderepilepsie. Zeitschrift ür Kinderheilkunde. Bd. 5, 1912. 